\title{
The Role of TREM-1 Gene Expression and Soluble TREM-1 as Prognostic Markers of Sepsis
}

\author{
Dina M Atef ${ }^{1 *}$, Rania A Ghonaim ${ }^{1}$ and Eslam $\mathbf{N}$ Nada $^{2}$ \\ ${ }^{1}$ Clinical Pathology Department, Faculty of Medicine, Zagazig University, Egypt \\ ${ }^{2}$ Anesthesia Department, Faculty of Medicine, Zagazig University, Egypt
}

\begin{abstract}
Background: Determination of TREM-1 expression on monocytes has been investigated as a perspective diagnostic method to distinguish infectious from non-infectious etiology of the inflammation.

Objectives: To analyse the expression of the TREM-1 gene in patients of sepsis and septic shock and its relation with the severity of pathophysiological conditions. Also to describe the dynamics changes of soluble TREM1 , procalcitonin and $\mathrm{C}$-reactive protein during the course of sepsis and Correlation between their expressions and the clinical scoring system known as APACHE II scoring system.

Material and Methods: After an ethical approval, the expression of mRNA of TREM-1 in PMNs was detected in 76 critically ill patients ( 49 with sepsis and 27 with septic shock) were analyzed by using quantitative real-time PCR. The concentration of s. TREM-1, CRP and PCT levels were measured by an enzyme immunoassay.

Results: On the ICU admission, the septic shock group displayed higher levels of sTREM-1, PCT, APACHE I score $(P<0.001)$ and $C R P(P=0.01)$ than the sepsis group. Significant positive correlation $(P<0.05)$ between APACHE II and both sTREM-1 and PCT in the prognosis of morbidity were shown. The mRNA levels of patients suffering from sepsis and septic shock were 0.54 - and 0.39 -fold lower compared to those of healthy subjects (1.06) respectively.

Conclusion: With regard to sepsis diagnosis and severity, sTREM-1 and PCT have positive values in prognostic assessment of the disease and may be taken as a survival-impacting risk factor. The TREM-1 gene expression levels isolated from patients with sepsis may be used as a surrogate marker for determining the severity.
\end{abstract}

\section{Keywords: APACHE; RT- PCR; Sepsis; TREM-1}

\section{Introduction}

Severe sepsis and septic shock are life-threatening complications of infections and the most common causes of morbidity and mortality among critically ill patients in the intensive care unit (ICU), blood culture has always been held as the gold standard for sepsis diagnosis [1]. However, treatment of these cases is delayed while waiting for lab results which may lead to worsen the condition of the patients, develop multiple organ dysfunction or failure and death for delayed ineffective treatment [2].

Triggering receptor expressed on myeloid cells-1 (TREM-1) was first described in the year 2000 [3], is a member of immunoglobulin (Ig) superfamily receptor expressed on polymorphonuclear granulocytes and mature monocytes, stimulation by bacteria or fungus infection may cause up-regulation for TREM-1 expression and transmit signals downstream, leads to production of pro-inflammatory mediators, mainly tumor necrosis factor-alpha and interleukin-8 and bring about relevant inflammatory responses [4]. TREM-1 is an amplifier of the immune response, which strongly potentiates the activation of leucocytes in response to microbial products [5].

A soluble form of TREM-1 (sTREM-1) is released from the activated phagocytes and can be found in body fluids of patients with sepsis and other inflammatory diseases caused by extracellular microorganisms such as pneumonia and cholangitis [6]. It is a superior indicators of sepsis compared to C-reactive protein (CRP) and procalcitonin (PCT) levels [7].

Additionally, clinical studies have shown that early diagnosis can significantly improve the prognosis of severe sepsis and septic shock and lower the mortality rate of the patients. Accordingly it becomes critical to discover new biomarkers that can accurately identify the cause being infectious or non-infectious together with predicting the prognosis [8].
Peripheral blood leucocytes constitute an accessible source of clinically relevant information and the gene expression profiling of these cells is an emerging strategy for proper diagnosing and monitoring of the infections. The gene expression patterns of TREM-1 existing in these cells of patients with bacterial infections has led to a better understanding of the principal mechanisms of infectious diseases and responses to treatment.

\section{Aim of the Study}

To study the effect of severity of infection on the expression of TREM-1 gene and its use as an early biomarker for determining the severity of sepsis in ICU patients. We also aimed to measure the serum level of TREM-1 in these patients, to find out its relation to clinical disease severity compared with other markers such as PCT and CRP and a clinical scoring system as APACHE score.

\section{Subjects and Methods}

\section{Subjects and samples collection}

To achieve this aim, the study was carried out on 100 individuals;

*Corresponding author: Dina M. Atef, Clinical Pathology Department Faculty of Medicine, Zagazig University, Egypt, Tel: 002-01002826410; E-mail: datefkareem@hotmail.com.

Received September 10, 2015; Accepted December 07, 2015; Published December 09, 2015

Citation: Atef DM, Ghonaim RA, Nada EN (2015) The Role of TREM-1 Gene Expression and Soluble TREM-1 as Prognostic Markers of Sepsis. J Med Microb Diagn 5: 211. doi:10.4172/2161-0703.1000211

Copyright: ( 2015 Atef DM, et al.. This is an open-access article distributed under the terms of the Creative Commons Attribution License, which permits unrestricted use, distribution, and reproduction in any medium, provided the original author and source are credited. 
they were classified into 76 critically ill patients $(47$ males and 29 females), who investigated twice after their admission to ICU and after treatment with proper antibiotics, and 24 apparently healthy individuals as a control group matched by sex and age with no signs of active inflammatory or infectious diseases (No fever, leukocytosis, redness, hotness or edema). The patients were chosen from those admitted to the Intensive Care Unit, Zagazig University Hospitals. Laboratory investigations were carried out at the Clinical Pathology Department, Zagazig University Hospitals during the period from Jun 2014/ Feb 2015.

Ethical consideration: A written consent was taken from all of the participants after explaining details, benefits as well as risks to them.

Inclusion and exclusion criteria: Based on the 2012 International Guidelines for Management of sever sepsis and septic shock [9] that advanced the diagnostic criteria, patients exhibiting two or more of the following signs during their first $24 \mathrm{~h}$ in the ICU were eligible for selection: (1) temperature of $>38.3^{\circ} \mathrm{C}$ or hypothermia $<36^{\circ} \mathrm{C}$, (2) pulse rate of $>90$ beats/min or more than two SD above the normal value for age, (3) respiratory rate of $>20$ breaths/min or hyperventilation with a partial pressure of arterial carbon dioxide $\left(\mathrm{PaCO}_{2}\right)$ of $<32 \mathrm{mmHg}$, (4) altered mental status, (5) significant edema or positive fluid balance ( $>$ $20 \mathrm{~mL} / \mathrm{kg}$ over $24 \mathrm{hr}$ ), (6) Hyperglycemia (plasma glucose $>140 \mathrm{mg} / \mathrm{dL}$ or $7.7 \mathrm{mmol} / \mathrm{L}$ ) in the absence of diabetes or (7) white blood cell (WBC) count of $>12,000 \mu \mathrm{L} / 1$ or $<4000 \mu \mathrm{L} / 1$, or $>10 \%$ immature cells.

Exclusion criteria were: (1) $<18$ years of age, (2) acquired immunodeficiency syndrome, (3) reduced polymorphonuclear granulocyte count $(<500 \mu \mathrm{L} / 1)$, or (4) died within $24 \mathrm{~h}$ after admission into the ICU, refused to participate in the study, or declined treatment during the observation period.

\section{Clinical and routine laboratory investigations}

Venous blood samples were taken from every patient and control person under complete aseptic conditions.

a. Serum CRP was measured using automated clinical chemistry analyzer Cobas Integra 400 plus (Roche Diagnostics, Deutschland).

b. PCT was measured using an enzyme-linked fluorescence analysis kit (ELFA, VIDAS BRAHMS PCT kit, bioMerieux SA, France).

c. The microorganism's growth in blood cultures was screened by the BacT/ALERT 3D Microbial Detection System (bioMérieux, Marcy l'Etoile, France) with aerobic (BacT/ALERT FA) and anaerobic (BacT/ ALERT FN) media. Further identification to the positive cultures using Matrix-assisted laser desorption ionization-time of flight mass spectrometry (MALDI-TOF MS) Vitek MS (bioMérieux, Marcy l'Etoile, France).

\section{Soluble TREM-1-analysis}

Measurement of plasma TREM-1 level by a double antibody sandwich ELISA (Enzyme-linked Immunosorbant assay) technique using Quantikine human TREM-1 Immunoassay ELISA Kit, from $\mathrm{R}$ \& D Systems, Minneapolis, MN, USA. The lower detection limit was $15.1 \mathrm{pg} / \mathrm{ml}$.

\section{TREM-1-gene expression}

We chose to study a relative quantitative value of TREM-1 CDNA to ACTB CDNA (Reference gene) in peripheral blood of patients suffering from sepsis based on their previously documented associations with bacterial infections $[7,10,11]$, ACTB was used as an endogenous control to detect the effect of hemolysis.
Gene expression level of TREM-1 in PMNs was quantified using the real-time PCR system. Complementary DNA was amplified with SYBR Green by using the Power SYBR Green PCR master mix (Jena Bioscience, Jena, Germany) according to the manufacturer's protocol. Each sample was duplicated and the mean of CT (Cycle Threshold) was calculated.

Briefly, qPCR was performed for TREM-1. PCR primer sequences were shown in Table 1.

\begin{tabular}{|c|c|c|}
\hline Genes & DNA Sequences & Amplicon sizes \\
\hline TREM-1 & $\begin{array}{l}\text { F: 5'-GTCTCCACTCCTGACTCTGAA-3' } \\
\text { R: 5'-TAGGGTACAAATGACTCTGAA-3' }\end{array}$ & $158 \mathrm{bp}$ \\
\hline \multirow{2}{*}{ ACTB } & $\begin{array}{l}\text { F: 5'-TTAAGGAGAAGCTGTGCTACG-3' } \\
\text { R: 5'-TTGAAGGTAGTTTCGTGGATG-3' }\end{array}$ & $205 \mathrm{bp}$ \\
\hline
\end{tabular}

Table 1: PCR primer sequences.

TREM-1 mRNA expression levels in PMNs were normalized to the gene expression levels of ACTB. Fold changes of PMN TREM-1 mRNA levels between patients and controls were calculated manually using the comparative cycle threshold $(\Delta \Delta \mathrm{Ct})$ method as follows: $\Delta \mathrm{Ct}=$ mean value $\mathrm{Ct}$ (cDNA of interest) - mean value $\mathrm{Ct}$ (reference ACTB), $\Delta \Delta \mathrm{Ct}=\Delta \mathrm{CT}$ test sample - Average $\Delta \mathrm{CT}$ control sample. The fold change in gene expression normalized to reference gene (R) of the sample and relative to the control, the negative value of this subtraction $(-\Delta \Delta \mathrm{CT})$ becomes the exponent of $2(\mathrm{R}=2-\Delta \Delta \mathrm{CT})$. Then the relative expression levels of TREM-1 cDNA were confirmed by using free data analysis tools.

\section{Statistical analysis}

The data was analyzed using SPSS statistical package software computer program version 17 (SPSS Inc., Chicago, IL). Statistical tests were applied to determine the relationships between TREM-1 gene expression, soluble TREM-1 concentrations, clinical and laboratory parameters in both ICU patients having sepsis and controls.

Quantitative data were presented as mean \pm standard deviation. Comparison of quantitative variables between the study groups was done using the Student ( $\mathrm{T})$ test. Qualitative data were expressed as frequency and percentage. Association between qualitative data was done using the chi-square test. Differences with $(\mathrm{P})$ values less than 0.05 were considered significant. $95 \%$ confidence interval was calculated to estimate the strength of the associations.

\section{Results}

A total of 76 patients with sepsis were included in this study. They manifested clinically as having fever which was the most common (95\%) then leucocytosis was represented in (89\%), then hyperglycemia was found in (32\% of patients group), these results were show in Table 2 . Clinical manifestations were presented at least once during the evolution period of the disease, measured from the time of diagnosis to the moment when a blood sample was drawn. 24 as a control group. Laboratory data of the patients and control groups were summarized in Table 2 .

Table 3 show assessment of CRP, PCT and Serum TREM-1 according to the sepsis diagnosis criteria (sepsis $(\mathrm{n}=49)$ septic shock $(\mathrm{n}=27))$. The bars indicate medians of s. TREM-1, PCT, and CRP levels between patients with sepsis and those with septic shock coming out as $114.8 \mathrm{pg} / \mathrm{mL}$ versus $367.6 \mathrm{pg} / \mathrm{mL}, \mathrm{P}<0.001 ; 7.23 \mathrm{ng} / \mathrm{mL}$ versus $22.17 \mathrm{ng} /$ $\mathrm{mL}, \mathrm{P}<0.0001$ and $23.8 \mathrm{mg} / \mathrm{dL}$ versus $29.4 \mathrm{mg} / \mathrm{dL}, \mathrm{P}=0.01$.

In Table 4 the APACHE II score showed significant difference $(\mathrm{p}<0.001)$ between sepsis $21.9 \pm 7.1$ and septic shock $28.2 \pm 7.8$. Also in Table 5 there was significant positive correlation between APACHE II 
and both procalcitonin and $\mathrm{s}$ TREM-1 but it was more positive for the later in the prediction of the severity of illness.

Fold changes of TREM-1 in sepsis patients (sepsis and septic shock) compared to the controls were shown in Table 6. As its expression is stable and reproducible, $A C T B$ was chosen as an endogenous control to standardize mRNA expression. We quantified the relative expression of the TREM- 1 in the PMNs of patients and control. The analysis revealed a highly significant down-regulation of TREM-1 in the form of 0.543 fold in sepsis patients $(\mathrm{P}<0.001)$, and 0.394 fold in patients with septic shock ( $\mathrm{P}=0.001$ ), when compared with the high level in controls being 1.061 fold. In contrast; there was less significant difference of TREM-1 expression between both sepsis and septic shock patients, $(\mathrm{P}<0.01)$.

\begin{tabular}{|c|c|c|c|c|}
\hline Characteristic & $\begin{array}{c}\text { Patients group } \\
\text { (76) }\end{array}$ & $\begin{array}{c}\text { Control group } \\
\text { (24) }\end{array}$ & $\mathbf{t}$ & $\mathbf{P}$ \\
\hline \multicolumn{5}{|l|}{ Atiological causes $(n, \%)$ : } \\
\hline Pulmonary Infection & $25(32.89 \%)$ & $0(0 \%)$ & & \\
\hline Abdominal Infection & $8(10.54 \%)$ & $0(0 \%)$ & & \\
\hline Urinary Infection & $11(14.47 \%)$ & $0(0 \%)$ & & \\
\hline Trauma/ post-operative & $17(22.36 \%)$ & $0(0 \%)$ & & \\
\hline Others & $15(19.74 \%)$ & $0(0 \%)$ & & \\
\hline Body temperature (fever) & $38.6 \pm 1.3$ & $37.2 \pm 0.5$ & 5.14 & $<0.001$ \\
\hline Hemoglobin (g/dl) & $9.1 \pm 1.7$ & $13.1 \pm 1.2$ & -5.34 & $<0.001$ \\
\hline WBCs $\left(\times 10^{3} / \mathrm{mm}^{3}\right)$ & $14.2 \pm 8.4$ & $5.1 \pm 2.2$ & 5.23 & $<0.001$ \\
\hline ESR 1st hour (mm/ hr.) & $33.6 \pm 21.7$ & $9.3 \pm 5.4$ & 5.41 & $<0.001$ \\
\hline Glucose $(\mathrm{g} / \mathrm{ml})$ & $148 \pm 69$ & $84 \pm 23$ & 4.45 & $<0.001$ \\
\hline
\end{tabular}

(Quantitative data of normal distribution are presented as mean \pm SD, Qualitative data are presented as $n(\%)$, Significant if $(P<0.05)$.

Table 2: Clinical and laboratory characteristics of patients and controls.

\begin{tabular}{|l|c|c|c|c|}
\hline & Sepsis (n= 49) & $\begin{array}{c}\text { Septic shock } \mathbf{( n} \\
\mathbf{=} \mathbf{2 7})\end{array}$ & T & P \\
\hline CRP $(\mathrm{mg} / \mathrm{dL})$ & $23.8 \pm 8.3$ & $29.4 \pm 10.7$ & 2.53 & 0.01 \\
\hline PCT $(\mathrm{ng} / \mathrm{mL})$ & $7.23 \pm 4.9$ & $22.17 \pm 5.4$ & 7.01 & $<0.001$ \\
\hline P.TREM-1 $(\mathrm{pg} / \mathrm{ml})$ & $114.8 \pm 23.5$ & $367.6 \pm 51.4$ & 29.41 & $<0.001$ \\
\hline
\end{tabular}

*Significant if $(P<0.05)$.

Table 3: Comparison of serum biomarkers and cytokines levels.

\begin{tabular}{|l|l|l|c|c|}
\hline & (Range) & $\mathbf{X} \pm$ SD & T & P \\
\hline Sepsis $(\mathrm{N}=49)$ & $(21-31)$ & $21.9 \pm 7.1$ & & \\
\hline Septic shock $(\mathrm{N}=27)$ & $(21.9-34)$ & $28.2 \pm 7.8$ & 14 & $<0.001$ \\
\hline
\end{tabular}

APACHE II score: acute physiologic assessment and chronic health evaluation II scores. Significant if $(P<0.05)$.

Table 4: APACHE II score in sepsis and septic shock.

\begin{tabular}{|l|c|c|c|}
\hline & r & P & Sig. \\
\hline Procalcitonin & 0.626 & 0.009 & S \\
\hline S. TREM-1 & 0.867 & 0.001 & S \\
\hline
\end{tabular}

Significant if $(P<0.05)$

Table 5: Correlation between APACHE II and both TREM-1 and procalcitonin in prognosis of morbidity.

(Spearman's Rho" correlation" calculator)

\begin{tabular}{|c|c|c|c|}
\hline & $\begin{array}{c}\text { Control group } \\
(\mathbf{n = 2 4 )}\end{array}$ & $\begin{array}{c}\text { Sepsis } \\
(\mathbf{n = 4 9 )}\end{array}$ & $\begin{array}{c}\text { Septic shock } \\
\mathbf{( n = 2 7 )}\end{array}$ \\
\hline TREM-1 gene & 1.061 & 0.543 & 0.394 \\
\hline $95 \% \mathrm{Cl}$ & $0.9-1.22$ & $0.389-0.697$ & $0.309-0.479$ \\
\hline \multicolumn{2}{|l}{$95 \% \mathrm{Cl}$ assuming equal variance. }
\end{tabular}

Table 6: The fold changes of TREM-1 patients compared to control group.

\section{Discussion}

Among phagocytes, PMNs are remarkable for their migratory capacity. Upon activation by chemoattractants, chemokines or inflammatory products, PMNs rapidly migrate to the sites of infection. Pathogen-associated molecular patterns (PAMPs) are recognized as molecular signatures by pattern recognition receptors (PRRs) that are predominantly expressed on PMN surface [12].

Sever sepsis and septic shock is common among critically ill patients, with reported mortality rates ranging from $25 \%$ to $70 \%$ when complicated by shock and multiple organ failure [2].

TREM-1 is a recently discovered member of the immunoglobulin superfamily of receptors that is specifically expressed on the surfaces of polymorphonuclear granulocytes and mature monocytes. Bacteria or fungi infections may up regulate expression, transmit signals downstream, induce the release of proinflammatory cytokines and bring relevant inflammatory responses [13].

A soluble form of TREM-1 (sTREM-1) that released into body fluids when TREM-1 is upregulated [13]. In a study done by Zhenyu et al., [14], they found that sTREM-1 levels of patients with septic shock were significantly higher than those with sepsis on first day of admission. 'In a study done by Ornatowska et al., [15] they proved that the levels of soluble TREM-1 in bronchoalveolar lavage fluid and serum of patients with pneumonia have shown to be an indicator and prognostic factor in sepsis and acute respiratory distress syndrome induced by microbial infections.

These findings come in agreement with our results, where the levels of sTREM-1 in patients with septic shock were significantly higher than those with sepsis being 367.6 versus $114.8 \mathrm{pg} / \mathrm{ml}$. Opposing our results prior studies by Phua et al., [16] and Bopp et al., [17], they failed to find the association between sTREM-1 and poor outcome.

Gibot and Cravoisy [18] reported that there is inverse correlation of TREM-1 mRNA levels with the severity of patho-physiological conditions of acute bacterial infections. Protein expression of TREM-1 is also up-regulated in phagocytic cells in the presence of pathogens and sTREM-1 is released into circulating blood during infection. These results come in the same line with our results, where the levels of TREM-1 mRNA decrease with severity of the disease. The levels in patients suffering from septic shock are less than those suffering from sepsis and both levels were less than that of the control.

In the same study done by Gibot and Cravoisy [18], the average expression levels of TREM-1 in moderate and sever sepsis patients were 1.10 and 0.35 -fold, respectively. In recovered patients TREM- 1 mRNA expression levels recovered to normal levels. Therefore, monitoring the mRNA levels of TREM-1 in the PMNs of patients could possibly reflect the effect of treatment.

This comes in agreement with our study, where the TREM-1 gene expression was down-regulated in septic patients ( 0.54 fold in sepsis and 0.39 with septic shock) compared to normal healthy controls (1.06 fold).

PCT is normally produced in the C cells of the thyroid glands, plasma PCT levels in healthy humans are approximately $5-50 \mathrm{pg} / \mathrm{ml}$ in normal state. Its half-time is about 22-33 hours in serum [19]. In study done by Christophe Clec'h et al., found that serum PCT on day 1 was significantly higher in patients with septic shock than those with sepsis. On addition among patients with sepsis, PCT concentrations were significantly higher in those who died than in the survivors at all four measurement time points [20]. Also in prognostic assessment of sepsis, PCT value was found to correlate significantly with the acute 
physiology and chronic health evaluation (APACHE II) [21]. In our study APACHE II was also significantly correlated with sTREM-1 more positively than procalcitonin.

CRP is an acute phase protein and a sensitive systemic marker of inflammation and tissue damage, its level increase within 4-6 h after stimulus, doubles every $8 \mathrm{~h}$ and peaks at 36-50 $\mathrm{h} \mathrm{[21].}$

Zhenyu et al., [14], showed no significant difference between survivors and non-survivors during the three measures. This indicates that CRP was just an inflammatory biomarker and failed in reflecting sepsis severity. Explaining that CRP rises slowly and peaks at $36 \mathrm{~h}$ after an endotoxin challenge Luzzani et al. [22], have reported that CRP levels in septic shock were lower than those in sepsis suggesting that CRP levels did not reflect the severity of sepsis.

Zhang et al. [23] suggested that serum sTREM-1 levels reflected the severity of sepsis more accurately than those of PCT and CRP and were more sensitive for dynamic evaluations of sepsis prognosis. It rises quickly, when exposed to infection and its half-time is short.

In a study done by Zhenyu et al. [14], they found that sTREM and PCT had an equal prognostic ability for sepsis mortality and were superior to other parameters. They reported that sTREM increases quickly, when exposed to infection and its half-time is short. In bacterial infections, serum PCT levels start to rise at $4 \mathrm{~h}$ after the onset of systemic infection and peak at between 8 and $24 \mathrm{~h}$, it decreased $50 \%$ every 24 hours along with therapy. These results come in agreement with our results, where the values of s. TREM and PCT proved to be more beneficial than the CRP levels. We also concluded that CRP is non-specific inflammatory marker and it doesn't reflect the severity of sepsis.

The prognostic difference may be dependent on their biologic and kinetic characteristics, where TREM-1 can stimulate neutrophil and monocyte-mediated inflammatory response via the triggering and release of pro-inflammatory cytokines and chemokines.

Our study, show the effect of severity of infection on the expression of TREM-1 gene for determining the severity of sepsis in ICU patients, and this is the first study in the Egyptian population.

Thus, we can conclude that TREM-1 is an attractive therapeutic target and a diagnostic marker for infectious inflammatory diseases. Thus, we can say that sTREM-1 is more ideal than PCT and CRP for early sepsis diagnosis and severity assessment and constitutes an independent risk diagnostic parameter.

Further work and future studies are needed together with increasing the sample size of patients. Also paying attention to the clinical care environment. All of these could help in applying these parameters in clinical decision making and improving the clinical outcome of patients having sepsis or those complicated with septic shock.

\section{Conflicts of Interest}

All authors declare no conflict of interest.

\section{References}

1. Weinstein MP, Towns ML, Quartey SM, Mirrett S, Reimer LG, et al. (1997) The clinical significance of positive blood cultures in the 1990s: a prospective comprehensive evaluation of the microbiology, epidemiology, and outcome of bacteremia and fungemia in adults. Clin Infect Dis 24: 584-602.

2. Shapiro MD, Howell DW, Bates DC, Angus L, Talmor D (2006) The association of sepsis syndrome and organ dysfunction with mortality in emergency department patients with suspected infection. Annals of Emergency Medicine 48: 583-590.

3. Bouchon A, Dietrich J, Colonna M (2000) Cutting edge: inflammatory responses can be triggered by TREM-1, a novel receptor expressed on neutrophils and monocytes. J. Immunol 164: 4991-4995.
4. Colonna M, Facchetti F (2003) TREM-1 (triggering receptor expressed on myeloid cells): a new player in acute inflammatory responses. Journal of Infectious Diseases 187: S397-S401.

5. Buckland KF, Ramaprakash H, Murray LA, Carpenter KJ, Choi ES, et al. (2011) Triggering receptor expressed on myeloid cells-1 (TREM-1) modulates immune responses to Aspergillus fumigatus during fungal asthma in mice. Immunol. Invest 40: 692-722.

6. Liao R, Liu Z, Wei S, Xu F, Chen Z, et al. (2009) Triggering receptor in myeloid cells (TREM-1) specific expression in peripheral blood mononuclear cells of sepsis patients with acute cholangitis. Inflammation 32: 182-190.

7. Gibot S, Cravoisy A, Kolopp-Sarda MN, Bene MC, Faure G, et al. (2005) Timecourse of STREM (soluble triggering receptor expressed on myeloid cells)-1, procalcitonin, and C-reactive protein plasma concentrations during sepsis. Crit Care Med 33: 792-796.

8. Dellinger RP, Carlet JM, Masur H, Gerlach H, Calandra T, et al. (2004) Surviving Sepsis Campaign guidelines for management of severe sepsis and septic shock. Crit Care Med 32: 858-873.

9. Dellinger RP, Levy MM, Rhodes A, Annane D, Gerlach H, et al. (2013) Surviving Sepsis Campaign: International Guidelines for Management of Severe Sepsis and Septic Shock: 2012. Crit Care Med 41: 58.0- 637.

10. Ubagai T, Nakano R, Kikuchi H, Ono Y (2014) Gene Expression Analysis of TREM1 and GRK2 in Polymorphonuclear Leukocytes as the Surrogate Biomarkers of Acute Bacterial Infections. Int J Med Sci 11: 215-221.

11. Dimopoulou I, Pelekanou A, Mavrou I, Savva A, Tzanela M, et al. (2012) Early serum levels of soluble triggering receptor expressed on myeloid cells-1 in septic patients: correlation with monocyte gene expression. J Crit Care 27: 294-300.

12. Kawai T, Akira S (2009) The roles of TLRs, RLRs and NLRs in pathogen recognition. Int Immunol 21: 317-337.

13. Bouchon A, Facchetti F, Weigand M, Colonna M (2001) TREM-1 amplifies inflammation and is a crucial mediator of septic shock. Nature 410: 1103-1107.

14. Zhenyu L, Hongxia W, Jian L, Bing C, Guangping L (2014) Serum soluble triggering receptor expressed on myeloid cells-1 and procalcitonin can reflect sepsis severity and predict prognosis: A prospective cohort study. Mediators of Inflammation 2014: 1-7.

15. Ornatowska M, Azim AC, Wang X, Christman JW (2007) Functional genomic of silencing TREM-1 on TLR4 signaling in macrophages. Am J Physiol Lung Cell Mol Physiol; 293: 1377-1384.

16. Phua J, Koay ESC, Zhang D, Lee KH (2008) How well doserum sTREM-1 measurements prognosticate in septic shock? Anaesthesia and Intensive Care 36: 654-658.

17. Bopp C, Hofer S, Bouchon A, Zimmermann JB, Martin E, et al. (2009) Soluble TREM-1 is not suitable for distinguishing between systemic inflammatory response syndrome and sepsis survivors and non survivors in the early stage of acute inflammation. European Journal of Anaesthesiology 26: 504-507.

18. Gibot S, Cravoisy A (2004) Soluble form of triggering receptor expressed on myloid cells-1 as a marker of microbial infection. Clin Med Res 2: 181-187.

19. Dahaba AA, Metzler H (2009) Procalcitonin's role in the sepsis cascade. Is procalcitonin a sepsis marker or mediator? Minerva Anestesiologica 75: 447-452.

20. Clech C, Ferriere F, Karoubi P, Fosse JP, Cupa M, et al. (2004) Diagnostic and prognostic value of procalcitonin in patients with septic shock. Critical Care Medicine 32: 1166-1169.

21. Endo S, Aikawa N, Fujishima S, Sekine I, Kogawa K, et al. (2008) Usefulness of procalcitonin serum level for the discrimination of severe sepsis: From sepsis; a multicenter prospective study. J Infect chemother 14: 244-249.

22. Luzzani A, Polati E, Dorizzi R, Rungatscher A, Pavan R, et al. (2003) Comparison of procalcitonin and C-reactive protein as markers of sepsis. Critical Care Medicine 31: 1737-1741.

23. Zhang J, She D, Feng D, Jia Y, Xie L (2011) Dynamic change of serum soluble triggering receptor expressed on myeloid cells-1 (sTREM-1) reflect sepsis severity and can predict prognosis: a prospective study. BMC Infectious Diseases 1: 53 . 